Nollner hat nachgewiesen, dass sich in Salpetermutterlaugen ein chlormagnesiumbaltiges Magnesiumborat abscheiden kann.

Ich nehme nun an, dass sich bei dem grossen Ueberschuss des Chlormagnesiums ein solches mit höherem Gehalt an Chlormagnesiumhydrat, als ihn der Boracit und dessen obige Formel aufweist, abschied.

Bei der Behandlung mit Wasser geht dieses jedoch unter allmählicher Abgabe jenes Ueberschusses in die stabilere Verbindung des Boracites über. Da wo im Salzlager eine nachträgliche Entziehung des Chlormagnesiumüberschusses statt hatte, trat diese Umbildung zu Boracit in krystallinischer Form auf, sobald die Kleinheit des Stassfurtit-Einschlusses eine genügende Einwirkung zuliess. In der That wurde zuerst B. Schultze auf kleine Krystalldrusen von Boracit aufmerksam, die nach innen immer kleinere Krystalle, im Centrum aber noch einen Kern unveränderten Stassfurtits enthielten. Dic Erscheinungen bei der Vorbereitung des Stassfurtits für die Analyse sprechen sehr für meine obige Ansicht; statt mit Wasser mulsste man denselben also mit gesättigter Chlormagnesiumlösung höchst fein reiben, dann Letztere abpressen und versuchen, ob Alkohol vielleicht den Rest ohne Zersetzung wegnähme.

\title{
Gehalt der Butter an Fettsäuren.
}

Von E. Reichardt in Jena.

Durch die Untersuchungen von Hehner und Reichert wurde zuerst die Aufmerksamkeit auf die Gleichmässigkeit der flüchtigen und festen Fettsäuren in der Butter hingelenkt und die Bestimmung derselben sehr bald als die entscheidende für die Erkennung der Unverfälschtheit einer Butter in Anspruch genommen.

Hehner ${ }^{1}$ verseift die wasserfreie, reine Butter mit Kali und und Alkohol, scheidet die festen Fettsäuren durch Säuren ab und bestimmt letztere mit grosser Vorsicht im völlig trockenen Zustande. Die Untersuchungen von Hehner ergaben Schwankungen von 85,4 bis 86,2 Proc. fester Fettsäuren; Turner fand bei ausschliesslicher Fütterung mit Oelkuchen 86,3 Proc.; ich fand (s. die gleiche Abhandl.

1) Diese Zeitschrift 1878. Bd. 212. S. 129 u. f. Zeitschr, für analytische Chemie 1877. S. 145. 
in d. Zeitschrift) $85,7-88,02$ Proc. oder im Mittel von 9 Versuchen 87,80 Proc. und Hehner sprach als Grenzzahl für reines Butterfett bis 88 Proc. fester Fettsäuren aus.

Unter den bekannten Fetten, welche zur Verfälschung der Butter dienen können, enthalten Rindstalg, Schweinefett, Gänsefett, Mohnöl, Mandelöl, Rüböl, Olivenöl 93,8-95,97 Proc. in Wasser unlöslicher Fettsäuren und geben dadurch genügend Anhalt zur Erkennung.

Fleischmann und Vieth ${ }^{1}$ fanden bei einer grossen Zahl Untersuchungen ächter Butter 85,79-89,73 Proc. in Wasser unloslicher Fettsäuren, jedoch letztere Zahl sehr selten und sprechen sich dahin aus, dass man bei 87,5 Proc. Gehalt und weniger sicherlich in den seltensten Fällen fehlgreifen dürfte, wenn man die Butter als ächt bezeichne, und schliessen sich der Grenzzahl 88 nach Hehner völlig an, als mit einem hohen Grad von Sicherheit versehen.

Dagegen fand Kretschmar ${ }^{2}$ mehrfach die Zahl über 89 und schlägt vor, die sog. Grenzzahl auf $90 \mathrm{zu}$ erhöhen; auch Jehn fand nach der Aenderung der Methode nach O. Dietsch (Zusammenschmelzen der Fettsäuren mit Wachs und dann erst Wiegen) 86,2 bis 89,0 Proc., die höheren Zahlen namentlich bei Zusatz von Wachs.

Eine wesentliche Verbesserung oder gegenüber zu stellende Methode gab nun Reichert ${ }^{3}$ durch Bestimmung der flüchtigen Fettsäuren, deren Menge durch die verbrauchten C.C. $1 / 10$ Normalalkali ausgedrückt wird.

„2,5 G. ${ }^{4}$ wasserfreies, durch Baumwolle filtrirtes und gereinigtes Fett werden im flüssigen Zustande in einem etwa $150 \mathrm{C}$.C. fassenden Kölbchen - am besten von der von Erlenme yer angegebenen Form - abgewogen, sodann $1 \mathrm{G}$. festes Kalihydrat und 20 C.C. 80 procentiger Weingeist hinzugefügt. Diese Mischung wird im Wasserbade unter fleissigem Umrühren so lange behandelt, bis die erhaltene Seife eine nicht mehr aufschäumende, schmierige Masse bildet. Hierauf werden 50 C.C. Wasser in das Kölbchen gegeben, und die Seife, nachdem sie sich im Wasser gelöst hat, mit $20 \mathrm{C}$. C. verdünter Schwefelsäure (1 C.C. reiner Schwefelsäure auf 10 C. C.

1) D. Zeitschr. 1878. Bd. 213. S. 257. Zeitschr. f. analyt. Chemie 1878. Bd. 17. S. 287.

2) D. Zeitschr. 1878. Bd. 213. S. 561. Chem. Bericht X. 2091.; fernor John, d. Zeitschr. Bd. 212. S. 335.

3) D. Z. 1879. S. 163 . Zeitschr. f. anal. Chemie 1879. S. 68.

4) Zeitschr. f. anal. Chemie 1879. S. 69. 
Wasser) zerlegt. Man unterwirft nun den Inhalt des Kölbchens der Destillation mit der Vorsicht, dass man, um das Stossen der Flüssigkeit zu vermeiden, einen schwachen Luftstrom durch letztere leitet. Auch empfiehlt es sich, eine Kugelröhre mit weiter Oeffnung, wie solche bei der Bestimmung des Siedepunktes angewandt wird, mit dem Kölbchen zu verbinden, um ein Ueberspritzen von Schwefelsäure zu verhutiten. Das Destillat, welches namentlich bei butterarmen Fetten und bei rascher Destillation stets etwas feste Fettsäuren absetzt, wird unmittelbar durch ein angefeuchtetes Papierfilter in ein 50 C. C. Fläschchen filtrirt. Nachdem etwa $10-20$ C. C. davon übergegangen, giesst man das Destillat wieder in das Kölbchen zurück und setzt hierauf die Destillation fort, bis das Fläschchen genau 50 C. C. Destillat enthält. Das Destillat, welches bei mässigem Gange der Destillation eine wasserhelle Flüssigkeit bleibt, wird sofort, nach Zusatz von 4 Tropfen Lackmustinktur, mit $1 / 10$ Normal-Natronlauge titrirt. Die Titration ist beendet, wenn die blaue Farbe des Lackmus auch nach längerer Zeit bestehen bleibt.“

Die Untersuchungen Reich $\theta$ rt's ergaben hierbei Schwankungen bei reinem Butterfett von 13,0-14,95 C.C. $1 / 10$ Normalalkali oder im Mittel 13,97, abgerundet 14,0 C.C.

Die gleich dargestellten Destillate von

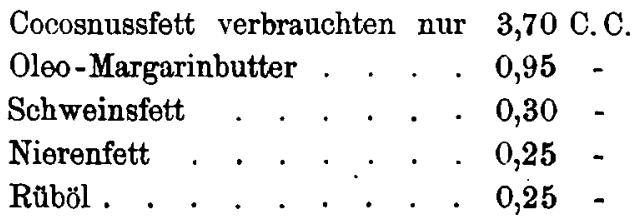

$1 / 10$ Normalalkali. Diese Unterschiede treten weit deutlicher hervor, und, da sie mittelst der leicht und sehr sicher auszuführenden Neutralisation mit titrirter Lauge erhalten werden, wird diese Methode die bruuchbarste, welche bis jetzt bekannt.

Um die Schwankungen der gefundenen Zahlen bei reinem Butterfette auszudrücken, giebt Reichert der Grenzzahl 14 noch das + oder $-0,45$, wobei allerdings die selbst von ihm erhaltenen höchsten Zahlen als Ausnahme nicht zur Geltung gelangen.

Die Controlbestimmungen mit künstlich dargestellten Fettgemischen entsprechen sehr gut den berechneten Zahlen und kann ich dies aus zahlreichen Untersuchungen meinerseits bestätigen.

Reichert glaubt daher nach seinen Untersuchungen nur Butter als ächt bezeichnen zu dürfen, welche mehr als 13 C.C. $1 / 10$ Normal- 
natronlauge erfordert, zu beanstanden aber bei Verbrauch von nur 12,5 C.C. Lauge u. s. w. Die Berechnungen künstlicher Gemische führen zu der allgemeinen Formel $B=a(n-b)$, wobei $B$ die Anzahl der Butterprocente, $n$ die verbrauchten C.C. Natronlauge bedeuten und die Gleichung ergiebt sich $=B=(7,30 \pm 0,24)(\mathbf{n}-0,30)$; hinsichtlich der Begründung ist die Originalarbeit einzusehen. Die Ausführung der Berechnung kann dann so geschehen: "Um den wahrscheinlichen Butterfettgehalt eines Fettgemisches in Procenten zu finden, ziehe man von der Anzahl der zur Titration verbrauchten Cubikcentimeter Natronlauge 0,30 ab und multiplicire den Rest mit 7,30."

Sieht man von den abzuziehenden 0,30 ab und berechnet man einfach den Verbrauch an C.C. Natronlauge in einfacher Proportion zu dem Verbrauche bei reiner Butter $=14$, so erhält man ebenfalls sehr nahe liegende Zahlen. Der Fehler steigt natürlich bei Zusatz geringer Mengen fremder Fette, was in der Regel nicht der Fall sein wird, um lohnenderen Gewinn zu erzielen.

Der Aufforderung Reichert's zu grösseren Controlversuchen folgte bald eine sehr ausführliche Arbeit von Meissl ${ }^{1}$ mit 84 verschiedenen Fettproben. Meissl verdoppelte das Gewicht der zu nehmenden Butter, statt 2,5 g. 5 g., und giebt das Verfahren folgend an:

„5 g. geschmolzenes, vom Bodensatz (Wasser, Salze, Caseïn) abgegossenes und filtrirtes Fett werden in einem, etwa $200 \mathrm{C} . \mathrm{C}$. fassenden Kölbchen mit $2 \mathrm{~g}$. festem Aetzkali (welches man sich als gleich lange Stückchen vorräthig hält) und 50 C.C. $70 \%$ tigen Alkohol unter Umschütteln im Wasserbade bis zum Verschwinden der oligen Tropfen erhitzt und die klare Seifenlösung sodann bis zur vollständigen Verflüchtigung des Alkohols eingedampft. Den erhaltenen dicken Seifenleim löst man in 100 C.C. Wasser und zersetzt ihn mit 40 C. C. verdünnter Schwefelsäure $(1: 10)$. Das Kölbchen wird nun mit einigen Hanfkorn-grossen Bimssteinstückchen beschickt, durch ein knieförmig gebogenes Kugelrohr mit einem kleinen Kühlapparat verbunden und der Inhalt, der ohne zu stossen ruhig kocht, der Destillation unterworfen; diese ist in etwa 1 Stunde beendet und liefert auch bei raschem Gange stets Schwefelsäure-freie Destil-

1) D. Zeitschr. 1879. Bd, 215. S. 531. Dingl. polytechn. Journ. Bd. 233. s. 229 . u. f. 
late. 110 C.C. des Destillates werden in einem entsprechend cubicirten Kölbchen aufgefangen, dann filtrirt und vom Filtrate $100 \mathrm{C}$ C. nach Zusatz einer immer gleichen, geringen Menge Lackmustinctur so lange mit $1 / 10$ Normal-Kalilauge versetzt, bis die blaue Farbe der Flüssigkeit auch nach längerem Schütteln sich nicht mehr verändert. Die Anzahl der verbrauchten C.C. Kalilauge muss selbstverständlich um $1 / 1_{0}$ vermehrt werden, um der Gesammtmenge des Destillates zu entsprechen. Der zu verwendende Alkohol soll frei von flüchtigen Säuren und Aldehyd, das Aetzkali nicht zu sehr mit Nitraten und Chloriden verunreinigt sein, um nicht aus diesen Quellen im Destillate freie Säuren zu erhalten. Aus diesem Grunde empfiehlt sich die Anstellung eines Controlversuches ohne Hinzuthun eines Fettes und muss derselbe ein säurefreies Destillat ergeben."

Meissl untersuchte hierbei ferner auch das Butteröl, d. h. den bei $20^{\circ} \mathrm{C}$. noch flüssig bleibenden Theil von geschmolzener und wieder erkalteter Butter, welcher durch Pressen getrennt wurde. Vier Proben selbst dargestelltes Butteröl verbrauchten 32,0 bis 34,5 C. C. $1 / 10$ Normalalkali im Destillate (oder nach Reichert halb soviel - 16,0-17,25 C.C. - , da Meissl die doppeite Menge Butter zur Prüfung nimmt), demnach sind in dem flüssigen Theile der Butter mehr flüchtige Säuren enthalten und werden weichere Buttersorten leicht etwas hohere Zahlen für das verbrauchte Alkali ergeben.

49 Proben ächter Butter verbrauchten $27-31,8$ C.C. Normalalkali (Reichert $=13,5-15,9$ ); bei nur 8 von den 49 Proben gebrauchte Meissl über 30 C.C. Normalalkali, bei weiteren 12 über 29 C.C. und die äbrigen 29 zwischen $27-29$ C.C. $(13,5-14,5)$, weshalb derselbe Butter, welche mehr als 27,0 C.C. $(13,5)$ Normalalkali verbraucht, als unzweifelhaft ächt ansehen will.

Die Ergebnisse Reicherts werden durch Meissl nur bestätigt und erweitert dahin, dass auch Erklärung für stärkeren Verbrauch an Alkali geschaffen wurde.

Die nach der Prüfung bekannter reiner Butterproben aufgeführten Ergebnisse bei Handelsware fallen sofort bedeutend herab, von $26,7-22,8$ C.C.

Selbst dargestellte Gemische 1) von $25 \%$ Schweineschmalz und und $75 \%$ Butter verbrauchten 22,2 C.C., 2) $35 \%$ Kunstbutter und 
$65 \%$ reiner Butter 20,0 C.C., 3) 50 Proc. reines Butterfett und 50 Proc. Talg und Schweineschmalz, 16,3 C. C., 4) $33 \%$ reines Butterfett $67 \%$ Rüböl und Talg, 11,6 C.C., 5) $25 \%$ reines Butterfett und $75 \%$ Schweineschmalz 9,6 C.C. und diesen Abstufungen entsprechen sehr gut die Handelssorten von Kunstbutter oder verschiedene Handelsbutterproben, welche als ächt in Wien angeboten wurden.

Diesen ausführlichen Arbeiten gegenüber fand Munier ${ }^{1}$ in Holland weit niedrigere Zahlen für den Verbrauch an Normalalkali nach Reichert; Munier ändert das Verfahren von Reichert abermals dahin ab, dass er zwar dieselbe Menge reines Butterfett nimmt, aber nur 5 C.C. einer 20procentigen alkoholischen Kalilösung, demnach $4 \mathrm{Mal}$ weniger Alkohol, wie Reichert. Munier giebt an, dass die grőssere Menge Alkohol unnöthig sei; vergleichende Versuche hier ergaben uns wiederholt weniger flüchtige Fettsäuren bei Verringerung des Alkohols, als ob bei dieser Aenderung die Verseifung doch nicht so vollständig erfolge. Es ist überhaupt nicht zu empfehlen, immer und immer Aenderungen eines Verfahrens zu bieten, ohne Mittheilung der Gegenversuche, welche genauestens beweisen müssen, dass dies neu geänderte Verfahren röllig gleiche Ergebnisse liefere. Munier entfernt dann die letzten Reste Alkohol durch Luftstrom und zersetzt die Seife mit Phosphorsäure statt Schwefelsäure.

Mit diesem so geänderten Verfahren verbraucht Munier bei ächter Butter bis nur 9,2 C.C. 1/10 Normalalkali und glaubt, namentlich eine verschiedene Mischung der Butter in verschiedener Jahreszeit annehmen zu müssen.

Für August bis October soll der durchschnittliche Verbrauch an Normalalkali 11 C.C. betragen, für October bis März 10,0 C.C., für März bis Mai 12,1 C.C. und für Mai bis August 12,4 C.C. Diese Zahlen sind sehr wesentlich niedriger, als diejenigen von Reichert und Meissl und Anderen, während bisher die Ergebnisse Reicherts nur bestätigt worden waren. Munier selbst zeigt auf Aenderung der Fütterung in den Jahreszeiten hin, auf Beginn der Weide, ohne bestimmte Ansprache zu erheben.

A mbuhl ${ }^{2}$ untersuchte Proben von Butter der Schweiz und verbrauchte nach Reichert im Mittel 14,67 C.C. Normalalkali, die

1) D. Zeitschr. 1882. Bd. 220. S. 850. Fresenius, Zeitschr. f. anal. Chemie 1882. S. 394.

2) D. Zoitschr. 1881. Bd. 218. S. 203. Schweizer. Wochenschrift f. Pharmacie 1881. S. 67. 
Mengen schwanken bei 6 verschiedenen Proben zwischen 14,05 bis 15,55 C.C., bestätigen demnach die Zahl Reicherts sehr gut und gleichzeitig die Versuche von Meissl, nach welchem dieser höhere Verbrauch auf flüssiges Butterfett zurückzufahren wäre.

Um diese für die Beurtheilung der Reinheit der Butter so wichtige Frage noch mehr zu klären, beschloss die hiesige Versuchsstation, eine Reihe dahingehender Versuche zu genehmigen. Herr Domänenpächter Graefe in Zwätzen bei Jena erklärte sich bereit, die Butterproben aus seinem Stalle zu liefern und Herr Assistent Koenig unternahm die Bestimmung, sowohl der in Wasser unloslichen Fettsäuren nach Hehner, wie der flüchtigen nach Reichert, in der ersten Zeit der Untersuchung sind beide Methoden nicht immer gleichzeitig in Verwendung gekommen. In dem Kammergute Zwätzen wird jetzt Holländer Raçe gehalten; der Wechsel des Futters wurde erst nach Beendigung der Versuche in Vergleich gezogen, um in keiner Weise auch nur äusserlich beeinflusst zu werden. Die Versuche ergaben :

\begin{tabular}{|c|c|c|c|c|}
\hline un & $\begin{array}{l}\text { flüchtig } \\
\text { osliche } \\
\text { nach H }\end{array}$ & $\begin{array}{l}\text { in Wasser } \\
\text { ettsäuren, } \\
\text { hn } \theta \mathrm{r}\end{array}$ & $\begin{array}{r}\text { flüchtige Fet } \\
\text { Reichert } \\
\text { mal }\end{array}$ & $\begin{array}{l}\text { tsäurer } \\
\text { in C.C } \\
\text { alkali }\end{array}$ \\
\hline 1882 & & & & \\
\hline 1. Dec. & 87,02 & Proc. & 14,3 & C. C. \\
\hline 8. - & 87,2 & - & - & - \\
\hline 15. - & 87,3 & - & - & - \\
\hline 22. - & 87,39 & - & - & - \\
\hline 29. - & 87,46 & - & - & - \\
\hline 5. Jan. & 87,37 & - & - & - \\
\hline 12. - & 88,30 & - & - & - \\
\hline 19. - & 90,0 & - & 13,8 & - \\
\hline 26. - & 88,5 & - & 14,4 & - \\
\hline 2. Eebr. & 87,29 & - & 14,4 & - \\
\hline 9. - & 87,32 & - & 14,6 & - \\
\hline 16. - & 87,3 & - & 14,7 & - \\
\hline 23. - & 87,4 & - & 14,5 & - \\
\hline 2. März & 87,7 & - & 14,0 & - \\
\hline 9. - & 87,4 & - & 14,3 & - \\
\hline 20. April & 87,7 & - & 14,0 & - \\
\hline 27. - & 87,2 & - & 14,3 & - \\
\hline 4. Mai & 87,25 & - & 14,0 & - \\
\hline 7. - & 87,40 & - & 14,4 & - \\
\hline
\end{tabular}


nicht flüchtige in Wasser
unlösliche Fettsäuren nach Hehner

1882

$\begin{array}{ccc}\text { 18. Mai } & 87,2 & \text { - } \\ \text { 25. - } & 87,4 & \text { - } \\ \text { 1. Juni } & 87,5 & \text { - } \\ \text { 8. - } & 87,7 & \text { - } \\ \text { 15. - } & 87,7 & \text { - } \\ \text { 22. - } & 87,6 & - \\ \text { 29. - } & 87,6 & - \\ \text { 6. Juli } & 87,3 & \text { - }\end{array}$

13. - 87,3 -

20. - 87,7 -

27. - 87,8 -

3. Aug. 87,4 -

10. - 87,8 -

17. - 87,4 -

24. - 87,5 -

31. - 87,7 -

7. Sept. 87,7 -

14. - 87,6 -

21. - 87,8 -

28. - 87,8 -

5. Oct. 87,6

12. - 87,6 -

19. - 88,0 -

26. - 87,6 - flüchtige Fettsäuren nach Reichert in C.C. Normalalkali

14,7 C.C.

13,9

13,9

14,0

14,1

13,8

13,9

14,4 -

13,9 -

$13,8-$

$14,7-$

$14,6-$

$14,0 \quad-$

13,9 -

$14,1 \quad-$

14,0 -

$14,3 \quad-$

$13,9-$

$13,8 \quad-$

14,3 -

14,0 -

$13,9-$

$3 \mathrm{Mal}$, d. 12., 19. und 26. Jan. wird die Zahl 88 bei den in Wasser unlöslichen Fettsäuren überschritten, die gleichzeitige Ermittelung der flüchtigen Fettsäuren am 19. und 26. Jan. hält sich aber völlig in den von Reichert gegebenen Grenzen von $14 \pm 0,45$. Man ist daher wohl veranlasst, hier Fehler in der Bestimmung zu suchen.

Das Mittel aller 43 Versuche ergiebt für die in Wasser unlöslichen Fettsäure 87,62 Proc. und lässt man die 2 höchsten Zahlen vom 12. und 19. Jan. fallen, so ändert dies so gut wie nichts, denn die Mittelzahl ist dann 87,55 .

Die Bestimmung der flüchtigen Fettsäuren schwankt zwischen 13,8-14,7 und das Mittel der 35 Versuche ist 14,16 C. C. $1 / 10$ Normalalkali. Bei dor Ermittelung der flüchtigen Fettsäuren wurde unter 
den 35 Bestimmungen nur 5 Mal die Zahl 14,5 um wenig äbersclıritten, 14,6 und 14,7. Die Schwankungen in sämmtlichen Bestimmungen lassen endlich in keiner Weise einen Einfluss der Jahreszeiten ersehen.

Nach dem nachträglich gegebenen Fütterungswechsel war derselbe gerade in der Bestimmungszeit ein sehr reger; hervorgerufen durch ortliche Verhältnisse, wie Separation, und durch die starke Trockenheit eines Theiles im Jahre.

Vom October bis gegen Mitte Mai wurde Winterfutter verabreicht für den Kopf Melkvieh 5 Pfund Weizenkleie (manchmal auch Roggenkleie), 2 Pfund Rapskuchen, 70 Pfund Runkelrüben, Gerstenstroh und Spreu (von allen Getreidearten gemengt) nach Belieben, im Durchschnitte 25 Pfund Stroh und Siede. Einige Tage in der Woche bekamen die Melkkühe für die Weizenkleie Biertrebern. In der Woche von 20-27. Mai wurde Mittags als Futter abgemähter Raps gefüttert; rom 28. Mai bis 4. Juni wurde auch Abends Grünfutter (Lupine) gegeben und von da zur alleinigen Grünfütterung übergegangen (Saufen nur reines Wasser). Ende Juni und die erste Hälfte des Juli wurden einige Zuckerrübenabschnitte schon mitgefüttert und dazu Hafer, Gerste und Erbsengemenge, im October nur Zucker - und Runkelrübenabschnitte. So weit die freundlichen Mittheilungen des Herrn Amtmann Gräfe.

In keiner Weise lassen diese ganz bedeutenden Aenderungen in der Nahrung, der Uebergang von Trocken- zu Grünfutter u. s. w. einen Einfluss auf die Mischung der Butter hinsichtlich der Fettsäuren erkennen. Weder Jahreszeit, noch Futterwechsel gestatten einen Anhalt zu irgend anderen Mittelzahlen, so dass diese Versuchsreihe nur das bestätigt, was Hehner wie Reichert von Anfang an erkannten und Meissl's zahlreiche Untersuchungen gleichfalls lehren, nämlich die annähernde Gleichheit der Butter in dem Gehalte der Fettsäuren.

Hehners erster Ausspruch, dass eine Butter, welche mehr als 88 Proc. im Wasser unlöslicher Fettsäure enthalte, zu beanstanden sei und zu anderweitigen Versuchen Anlass gebe, bestätigt sich hier völlig und ebenso die von Meissl gezogenen Sätze für die flüchtigen Fettsäuren, dass eine Butter zu beargwohnen sei, welche weniger als 13 C.C. $1 / 10$ Normalalkali zur Neutralisation der flüchtigen Fettsäuren bedürfe.

Beide Bestimmungen der in Wasser unlöslichen Fettsäuren und vielleicht noch mehr diejenige der flüchtigen geben den sichersten 
Anhalt zur Beurtheilung einer Butter, jedoch ist es bei der auszusprechenden Beschnldigung jedenfalls angemessen, noch einige, weitere Prüfungen zur Bestätigung des Verdachtes anzustellen, sowie auch anderweitige Zusätze festzustellen.

Ambuhl erwähnt in seiner oben besprochenen Arbeit über schweizerische Butter die mikroskopische Prüfung, Bestimmung des Fettgehaltes, des specifischen Gewichtes des Butterfettes bei $100^{\circ} \mathrm{C}$, die Prïfung auf Farbstoffe. Ich erlaubte mir, vor dem Bekanntwerden der Untersuchungsweisen von Hehner und Reichert in dieser Zeitschrift 1877. Bd. 210. S. 339 eine Uebersicht der bekannten Verfälschungen der Butter zu geben, mit der Erkennungsweise, wobei namentlich auch die Bestimmung des Schmelzpunktes von Butter und der Fettsäuren hervorgehoben wurde. Diese Untersuchungen haben mir bis jetzt stets röllig sichere Unterlage zur Beurtheilung einer fraglichen Butter gewährt.

Auch die von Birnbaum jüngst herausgegebene Prüfung der Nahrungsmittel im Grosherzogthum Baden bestätigt die Angaben von Munier in keiner Weise; die von ihm geleiteten Untersuchungen von Butterproben ergaben niemals unter $13 \mathrm{C}$. C. Normalalkali nach Reichert, auch nicht bei Prüfungen in den verschiedenen Monaten und so müssen diese Ergebnisse von Munier erst weiter bestätigt werden, wenn nicht, wie früher erwähnt, die bedeutende Verminderung des Alkohols bei der Verseifung die alleinige Schuld trägt, wie Versuche von uns es mehrfach ergaben.

Die Beobachtungen in Deutschland, auch an fremdländischer Butter, haben stets Bestätigungen der oben mehrfach wiederholten Zahlen von Reichert und Hehner ergeben.

\section{B. Monatsbericht.}

Zur Prïfung des Natrium benzoleum. - Nachdem Hell wig auf die Inconsequenz hingewiesen hatte, welche darin liegt, dass die Pharmacopöe eine aus Siamharz durch Sublimation bereitete Benzoësäure verlangt, für Natrium benzoicum eine gleiche Bestimmung aber nicht getroffen hat, was um so mehr zu bedauern sei, als erfahrungsgemäss das mit ächter Harzbenzoësäure hergestellte, freilich auch sehr theure Natriumbenzoat eine kräftigere und bessere Wirkung habe, als das mit künstlicher Säure bereitete, - theilte Hager mit, dass bezüglich der Unterscheidung beider Präparate 